\title{
Anthracimycin activity against contemporary methicillin-resistant Staphylococcus aureus
}

\author{
Mary E Hensler ${ }^{1}$, Kyoung Hwa Jang ${ }^{2}$, Wdee Thienphrapa ${ }^{1}$, Lisa Vuong ${ }^{1}$, Dan N Tran ${ }^{1}$, Evaristus Soubih ${ }^{1}$, \\ Leo Lin ${ }^{1}$, Nina M Haste ${ }^{3}$, Mark L Cunningham ${ }^{4}$, Bryan P Kwan ${ }^{4}$, Karen Joy Shaw ${ }^{4}$, William Fenical ${ }^{2}$ and \\ Victor Nizet ${ }^{1,3}$
}

Anthracimycin is a recently discovered novel marine-derived compound with activity against Bacillus anthracis. We tested anthracimycin against an expanded panel of Staphylococcus aureus strains in vitro and in vivo. All strains of $S$. aureus tested, including methicillin-susceptible, methicillin-resistant (MRSA) and vancomycin-resistant strains of $\boldsymbol{S}$. aureus, were susceptible to anthracimycin at MIC values of $\leqslant 0.25 \mathrm{mg} \mathrm{I}^{-1}$. Although its postantibiotic effects were minimal, anthracimycin exhibited potent and rapid bactericidal activity, with a $>4-\log$ kill of USA300 MRSA within $3 \mathrm{~h}$ at five times its MIC. At concentrations significantly below the MIC, anthracimycin slowed MRSA growth and potentiated the bactericidal activity of the human cathelicidin, LL-37. The bactericidal activity of anthracimycin was somewhat mitigated in the presence of $20 \%$ human serum, and the compound was minimally toxic to human cells, with an $\mathrm{IC}_{50}$ (inhibitory concentration 50) $=70 \mathrm{mgI}^{-1}$ against human carcinoma cells. At concentrations near the MIC, anthracimycin inhibited $S$. aureus nucleic acid synthesis as determined by optimized macromolecular synthesis methodology, with inhibition of DNA and RNA synthesis occurring in the absence of DNA intercalation. Anthracimycin at a single dose of 1 or $10 \mathrm{mg} \mathrm{kg}^{-1}$ was able to protect mice from MRSA-induced mortality in a murine peritonitis model of infection. Anthracimycin provides an interesting new scaffold for future development of a novel MRSA antibiotic.

The Journal of Antibiotics (2014) 67, 549-553; doi:10.1038/ja.2014.36; published online 16 April 2014

Keywords: anthracimycin; methicillin-resistant; novel antibiotic; Staphylococcus aureus

\section{INTRODUCTION}

The continued prevalence of methicillin-resistant Staphylococcus aureus (MRSA) infections represents a major clinical challenge in the United States. ${ }^{1,2}$ Complicating this fact, the rate of new antibiotic discovery over the past decade has not nearly kept pace with the rapid development of antibiotic resistance. ${ }^{3}$ Discovery of novel chemical entities with potent antibiotic activities is critical to continue the pipeline of therapeutic development. Marine-derived bacteria represent an important source of new chemical scaffolds with antiMRSA activity. ${ }^{4-8}$ A novel tricyclic dione, termed anthracimycin, was recently purified from a previously uncharacterized marine-derived species of Streptomycetes and was found to have potent activity against Bacillus anthracis and other Gram-positive bacteria. ${ }^{9}$ However, its activity against clinically relevant drug-resistant pathogens including MRSA is unknown. Therefore, we undertook a more comprehensive analysis of anthracimycin activity in vitro and in vivo.

\section{MATERIALS AND METHODS}

Isolation of anthracimycin

Anthracimycin was purified exactly as described from the marine-derived Streptomyces strain $\mathrm{CNH} 365,{ }^{9}$ and the structure of the purified compound was determined by combined spectroscopic methods and confirmed by a single crystal X-ray experiment (Figure 1). ${ }^{9}$

\section{MIC assays}

Antimicrobial activity was evaluated by broth microdilution according to CLSI guidelines (Clinical and Laboratory Standards Institute, 2009. M100-S19 Methods for Dilution Antimicrobial Susceptibility Tests for Bacteria that Grow Aerobically; approved standard, 9th edn. CLSI: Wayne, PA). MIC values in the presence of human serum were determined as described previously ${ }^{10}$ by the addition of $20 \%$ pooled human serum (collected from $>5$ donors according to an approved UCSD Institutional Review Board protocol). Bacterial viability at the end of the assay was determined by the addition of resazurin to the wells. The MIC in the serum was then determined to be the lowest compound concentration that did not produce a visible conversion of the resazurin (blue color) to resorufin (pink color). ${ }^{10}$

\section{Time-kill kinetics}

Anthracimycin time-kill kinetics and postantibiotic effects were performed in duplicate by broth macrodilution. For the time-kill kinetics, anthracimycin was added to CA-MHB (cation-adjusted Mueller-Hinton broth) at $0 \times, 1 \times, 5 \times$, $10 \times$ or $20 \times$ MIC $\left(\mathrm{MIC}=0.125 \mathrm{mgl}^{-1}\right.$ for USA300 MRSA strain TCH1516) in duplicate sterile polystyrene tubes (Falcon, Bedford, MA, USA). The media

\footnotetext{
${ }^{1}$ Department of Pediatrics, University of California San Diego, La Jolla, CA, USA; ${ }^{2}$ Center for Marine Biotechnology and Biomedicine, Scripps Institution of Oceanography, University of California San Diego, La Jolla, CA, USA; ${ }^{3}$ Skaggs School of Pharmacy and Pharmaceutical Sciences, University of California San Diego, La Jolla, CA, USA and ${ }^{4}$ Trius Therapeutics, San Diego, CA, USA

Correspondence: Dr ME Hensler, Department of Pediatrics, University of California San Diego, 9500 Gilman Drive, MC0760, La Jolla, CA 92093-0760, USA.

E-mail: mhensler@ucsd.edu
}

Received 4 December 2013; revised 18 February 2014; accepted 27 February 2014; published online 16 April 2014 
were then inoculated with $\sim 5 \times 10^{5} \mathrm{CFUs} \mathrm{ml}^{-1}$ in a final volume of $5 \mathrm{ml}$, and the tubes were incubated in a shaking incubator (New Brunswick, Enfield, CT, USA) at $37^{\circ} \mathrm{C}$. Viable bacteria over time were quantitated by the removal of $25 \mu \mathrm{l}$ aliquots for serial dilution in phosphate-buffered saline and plating on Todd-Hewitt agar (Hardy Diagnostics, Santa Maria, CA, USA). Time-kill kinetic studies were performed in triplicate.

\section{Postantibiotic effect}

MRSA strain TCH1516 was inoculated into $5 \mathrm{ml}$ of CA-MHB containing anthracimycin $\left(\mathrm{MIC}=0.125 \mathrm{mgl}^{-1}\right)$ or vancomycin $\left(\mathrm{MIC}=0.78 \mathrm{mgl}^{-1}\right)$ at $1 \times$ or $10 \times \mathrm{MIC}$ and incubated in a shaking incubator at $37^{\circ} \mathrm{C}$. At $1 \mathrm{~h}$, the bacteria were pelleted, washed two times in $10 \mathrm{ml}$ of phosphate-buffered saline and then resuspended in $4 \mathrm{ml}$ CA-MHB. The tubes were placed back in the $37^{\circ} \mathrm{C}$ shaking incubator, and bacterial regrowth was measured at selected timepoints up to $24 \mathrm{~h}$ by plating $25 \mu \mathrm{l}$ samples of serial dilutions on ToddHewitt agar plates and counting colonies.

\section{MRSA growth in sub-MIC anthracimycin}

Growth curves at sub-MIC concentrations of anthracimycin, vancomycin or vehicle control were performed by broth macrodilution format. Duplicate glass tubes containing CA-MHB ( $5 \mathrm{ml}$ per tube) with either anthracimycin or vancomycin at predetermined concentrations were inoculated with $\sim 5 \times 10^{5}$ CFU ml ${ }^{-1}$ MRSA strain TCH1516 and incubated in a shaking incubator at $37^{\circ} \mathrm{C}$. Growth was monitored at various timepoints up to $24 \mathrm{~h}$ by measuring absorbance at $600 \mathrm{~nm}$ in a spectrophotometer (Spectronic 20D +; Thermo Scientific, Waltham, MA, USA).

Growth studies at sub-MIC anthracimycin and LL-37 were performed in broth microdilution format in 96-well plates using RPMI supplemented with $5 \%$ Luria broth. RPMI supplemented with $5 \%$ Luria broth $(0.2 \mathrm{ml})$ containing $1 / 2 \times$ MIC of either: LL-37 (LL-37 MIC in RPMI supplemented with 5\% Luria broth $=16 \mu \mathrm{M}$ ), anthracimycin, LL-37 in combination with anthracimycin or vehicle was inoculated with $\sim 5-10 \times 10^{5} \mathrm{CFU} \mathrm{ml}^{-1} \mathrm{MRSA}$ strain TCH1516. The plates were incubated at $37^{\circ} \mathrm{C}$, and bacterial survival over time was monitored by plating $25 \mu \mathrm{l}$ serial dilutions on Todd-Hewitt agar for quantitative cultures.

\section{Mammalian cell cytotoxicity}

We assessed mammalian cytotoxicity using the human cervical carcinoma line HeLa (CCL-2, American Type Culture Collection, Manassas, VA, USA). HeLa cells were plated at a density of $2 \times 10^{4}$ cells per well of sterile flat-bottom 96well tissue-culture-treated plates (Corning-Costar, Corning, NY, USA), and anthracimycin was added at increasing concentrations. The plates were incubated in $5 \% \mathrm{CO}_{2}, 37^{\circ} \mathrm{C}$ and cell viability (proliferation) was analyzed at $72 \mathrm{~h}$ using the Promega Cell Proliferation Kit (Promega, Madison, WI, USA) according to the manufacturer's instructions, and the $\mathrm{IC}_{50}$ (inhibitory concentration 50) was determined.

\section{Mechanism of action studies}

Anthracimycin mechanism of action studies were carried out using an optimized macromolecular synthesis assay. ${ }^{11}$ Briefly, S. aureus strain ATCC

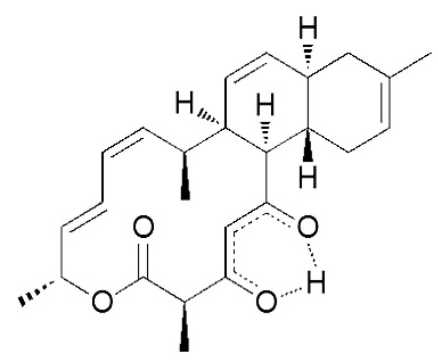

Figure 1 Chemical structure of anthracimycin. A single crystal X-ray experiment was used to confirm the structure of anthracimycin as determined by combined spectroscopic methods.
29213 was grown to mid-logarithmic phase in CA-MHB. Macromolecular synthesis in the presence of increasing doses of anthracimycin was monitored by measuring the incorporation of radiolabeled precursors of DNA, RNA, protein and cell wall synthesis $\left(\left[{ }^{3} \mathrm{H}\right]\right.$ thymidine, $\left[{ }^{3} \mathrm{H}\right]$ uridine, $\left[{ }^{3} \mathrm{H}\right]$ leucine and $\left[{ }^{3} \mathrm{H}\right]-\mathrm{N}$-acetylglucosamine, respectively) in the presence of increasing anthracimycin. The extent by which synthesis of each pathway was inhibited was determined by calculating the difference in label incorporation relative to untreated controls. DNA intercalation was examined using a commercially available kit (TopoGen, Port Orange, FL, USA) and observation of the products by agarose gel electrophoresis.

\section{Murine infection studies}

For the in vivo infection studies, 8-week-old female CD1 mice (Charles River Laboratories, Wilmington, MA, USA) were inoculated intraperitoneally with $\sim 1 \times 10^{9} \mathrm{CFU}$ of MRSA strain Sanger 252 (a sequenced hospital-associated MRSA strain) and then treated $1 \mathrm{~h}$ later with a single i.p. dose of anthracimycin ( 1 or $10 \mathrm{mg} \mathrm{kg}^{-1}$ ) or equivalent vehicle control ( $n=10$ mice per group). Survival was monitored over 7 days, and mice that appeared moribund were humanely killed. This in vivo infection study was performed in duplicate, and data from one representative experiment are shown. All studies involving animals were reviewed and approved by the University of California San Diego Animal Care and Use Committee.

\section{RESULTS}

We found that anthracimycin was potent against all strains of $S$. aureus tested, including methicillin-susceptible and -resistant strains and vancomycin-resistant $S$. aureus, with MIC $\leqslant 0.25 \mathrm{mgl}^{-1}$ (Table 1). Anthracimycin was also active against clinical isolates of vancomycin-resistant Enterococcus faecalis. The potent Gram-positive activity was, however, mitigated in the presence of $20 \%$ human serum, which resulted in significant increases in the MIC against

\section{Table 1 MIC values}

\begin{tabular}{|c|c|}
\hline Strain & $M I C\left(m g I^{-1}\right)$ \\
\hline \multicolumn{2}{|l|}{ MRSA } \\
\hline Sanger 252 (USA200) & 0.063 \\
\hline TCH1516 (USA300) & 0.125 (16 in $20 \%$ serum) \\
\hline UAMS1182 (USA300) & 0.125 \\
\hline ATCC 33591 & 0.125 \\
\hline NRS70 (ST5) & 0.08 \\
\hline NRS100 (ST250) & 0.08 \\
\hline NRS192 (ST1) & 0.16 \\
\hline VRSA-PA & 0.125 \\
\hline VRSA-MI & 0.25 \\
\hline \multicolumn{2}{|l|}{ Other S. aureus } \\
\hline UAMS1 & 0.125 ( 8 in $20 \%$ serum) \\
\hline NRS77 (sequenced ST8) & 0.16 \\
\hline NRS135 (ST8) & 0.16 \\
\hline RN4220 & 0.125 (32 in $20 \%$ serum) \\
\hline Newman & 0.16 \\
\hline \multicolumn{2}{|l|}{ Non-S. aureus } \\
\hline Enterococcus faecalis (ATCC 51299) & 0.25 \\
\hline E. faecalis isolate 6981 & 0.125 \\
\hline Bacillus anthracis (Sterne) & 0.03 \\
\hline Moraxella catarrhalis (ATCC 25238) & 4 \\
\hline Pseudomonas aeruginosa (ATCC 27853) & $>64$ \\
\hline Klebsiella pneumoniae (ATCC 700603) & $>64$ \\
\hline Acinetobacter baumannii (ATCC 17978) & $>64$ \\
\hline
\end{tabular}

Abbreviation: MRSA, methicillin-resistant $S$. aureus. 
MRSA and methicillin-sensitive S. aureus (Table 1). Although this compound was active against the Gram-negative pathogen, Moraxella catarrhalis ( $\mathrm{MIC}=4 \mathrm{mgl}^{-1}$ ), other clinically-significant Gram-negative organisms including Klebsiella pneumoniae and Acinetobacter baumannii were not susceptible to anthracimycin, with MIC > $64 \mathrm{mgl}^{-1}$ for these strains.

Anthracimycin exhibited rapid killing kinetics, with a $>4$-log kill of USA300 MRSA within $3 \mathrm{~h}$ at $\geqslant 5 \times$ MIC (Figure 2a). Despite these rapid killing kinetics, anthracimycin showed minimal postantibiotic effects against USA300 MRSA, with regrowth occurring rapidly after removal of the compound (Figure 2b). Mammalian cell cytotoxicity over $72 \mathrm{~h}$ yielded an $\mathrm{IC}_{50}=70 \mathrm{mgl}^{-1}$, which is several fold higher than the serum MIC of $16 \mathrm{mgl}^{-1}$ for USA300 MRSA. Given the rapid killing kinetics, we investigated the effects of subinhibitory concentrations of anthracimycin on USA300 MRSA. Even at $1 / 16 \times$ MIC $\left(0.0078 \mathrm{mgl}^{-1}\right)$, anthracimycin substantially reduced the growth rate of MRSA during the first $8 \mathrm{~h}$ of its logarithmic growth phase (Figure 3a). Comparatively, the cell wall antibiotic vancomycin had no effect on MRSA growth at $1 / 2 \times \operatorname{MIC}\left(0.78 \mathrm{mgl}^{-1}\right.$; Figure $\left.3 \mathrm{a}\right)$. Although no interactions with other commonly prescribed MRSA antibiotics including vancomycin and daptomycin were detected in traditional checkerboard assays, we tested anthracimycin for interactions with a key constituent of the host immune defense, cathelicidin antimicrobial peptides. For these studies MRSA was coincubated with sub-MIC concentrations of both anthracimycin and the human cathelicidin LL-37, and bacterial survival was monitored at selected time points by plating on agar. The addition of $1 / 4 \times$ MIC anthracimycin reduced viable MRSA counts in the presence of $1 / 2 \times$ MIC of LL-37 compared with either anthracimycin or LL-37 alone (Figure $3 \mathrm{~b}$ ). Additional studies indicated that this potentiation of activity was significantly reduced in the presence of $20 \%$ human serum (data not shown).

Given the potent in vitro activity of anthracimycin, we investigated its mechanism of action in S. aureus using an optimized macromolecular synthesis assay to quantitate incorporation of radiolabeled precursors. ${ }^{11}$ The primary effect was on $\left[{ }^{3} \mathrm{H}\right]$ thymidine and $\left[{ }^{3} \mathrm{H}\right]$ uridine incorporation, indicating the disruption of DNA and RNA synthesis, respectively (Figure 4). Disruption of these pathways occurs around the MIC concentration, suggesting that this effect is likely related to the antibacterial mechanism of action. Additional secondary effects on protein synthesis were also observed at much higher concentrations, at least 10-fold higher than the MIC. Primary disruption of DNA and RNA synthesis in the macromolecular synthesis assay is often associated with DNA intercalators. However, when anthracimycin was examined for evidence of DNA intercalation over the same concentration range as used in the macromolecular synthesis assay, no effect on DNA migration was observed relative to the untreated control (relaxed plasmid). Concentrations of $128 \mathrm{mgl}^{-1}, 1000$-fold higher than the MIC, show no evidence of DNA intercalation (Figure 4). These data suggest that the disruption of DNA and RNA synthesis by anthracimycin is not due to DNA intercalation.

To ascertain potential in vivo efficacy of anthracimycin, we tested its ability to protect mice from MRSA-induced mortality in a peritonitis model. Female CD1 mice were first infected intraperitoneally with $\sim 10^{9} \mathrm{CFU}$ of MRSA strain Sanger 252, a route and inoculum producing rapid bacteremia and subsequent lethality. One hour after infection, the mice were divided into three groups ( $n=10$ per group) and received a single i.p. injection of either anthracimycin (1 or $10 \mathrm{mg} \mathrm{kg}^{-1}$ ) or equivalent vehicle control; anthracimycin at these doses appeared to be well tolerated by the mice. Anthracimycin at either dose injected after infection was sufficient to provide significant protection against mortality over 7 days compared with infected mice treated with vehicle control alone (Figure 5). These results suggest that anthracimycin retained anti-MRSA activity and was also well tolerated when introduced in vivo.

\section{DISCUSSION}

The dearth of new chemical entities targeting problematic pathogens such as $S$. aureus has created a vacuum in the availability of novel antibiotics to treat these multidrug-resistant pathogens, and new antibiotics are urgently needed. The marine environment has been probed for decades as a source for novel scaffolds targeting cancer, however, similar studies targeting infectious diseases are rare. The purification of anthracimycin from a marine-derived species of Streptomycetes and initial identification of its Gram-positive activity prompted us to explore further the potential of this scaffold as an anti-MRSA compound. Our results show that anthracimycin activity extends across 14 strains of $S$. aureus tested, including USA300 strains of MRSA, vancomycin-resistant $S$. aureus and methicillin-sensitive $S$. aureus. No significant Gram-negative activity was observed. Human serum significantly reduced but did not completely eliminate anthracimycin activity. Despite this serum-induced loss of activity, a single dose of anthracimycin was still protective in the murine peritonitis
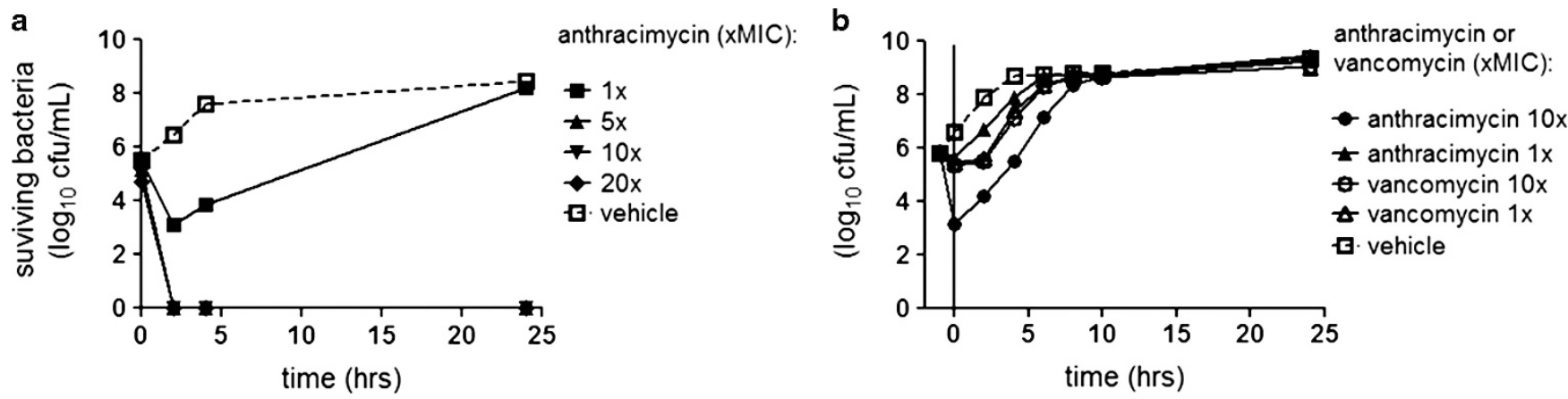

Figure 2 Kinetics of anthracimycin activity against USA300 methicillin-resistant S. aureus (MRSA) (strain TCH1516). (a) Time-kill kinetics of anthracimycin at $1 \times, 5 \times, 10 \times$ or $20 \times \mathrm{MIC}\left(\mathrm{MIC}=0.125 \mathrm{mgI}^{-1}\right)$ during $24 \mathrm{~h}$ incubation. (Note that the data points for the $5 \times, 10 \times$ and $20 \times \mathrm{MIC}$ anthracimycin are superimposed on the graph.) (b) Postantibiotic effect of anthracimycin against USA300 MRSA (strain TCH1516) compared with vancomycin. The postantibiotic effect was measured by incubating MRSA with anthracimycin or vancomycin for $1 \mathrm{~h}$ (vertical line at $0 \mathrm{~h}$ on the graph) and assessing $24 \mathrm{~h}$ regrowth (viable bacteria) after washing the bacteria to remove the antibiotics. (Note that the data points for the $1 \times$ and $10 \times$ MIC vancomycin are nearly superimposed on the graph.) 

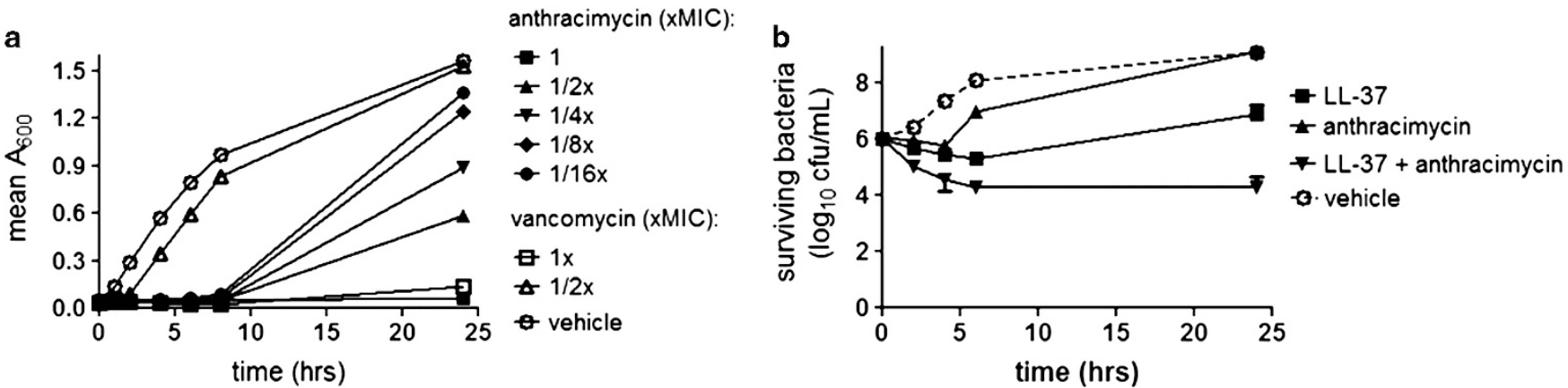

Figure 3 Effects of sub-MICs of anthracimycin on USA300 methicillin-resistant $S$. aureus (MRSA) growth and antimicrobial peptide sensitivity. (a) Growth curves of MRSA (strain TCH1516) in the presence of vehicle or increasing sub-MICs of anthracimycin $\left(\mathrm{MIC}=\left.0.125 \mathrm{mg}\right|^{-1}\right.$ ) compared with $1 / 2 \times$ or $1 \times$ MIC vancomycin (vancomycin $\mathrm{MIC}=0.78 \mathrm{mgl}^{-1}$ ). Turbidity as a measure of bacterial growth was monitored by OD $\left(A_{600}\right)$ over time in duplicate tubes. (b) Killing of MRSA strain TCH1516 during incubation with: vehicle (MRSA alone), 1/2 $\times$ MIC LL-37 alone, $1 / 4 \times$ anthracimycin alone or $1 / 2 \times$ MIC LL-37 +1/4 $\times$ MIC anthracimycin. Surviving bacteria were enumerated by plating on agar, and the data represent mean \pm s.d. of duplicate samples.
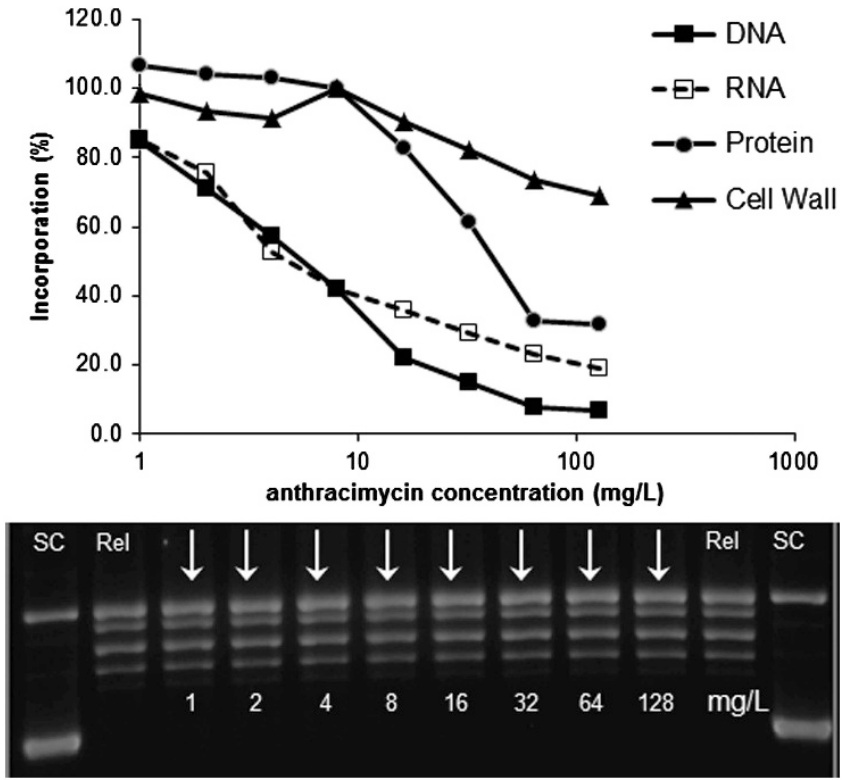

Figure 4 Macromolecular synthesis in the presence of increasing anthracimycin. Incorporation of radiolabeled precursors of DNA, RNA, protein and cell wall synthesis $\left(\left[{ }^{3} \mathrm{H}\right]\right.$ thymidine, $\left[{ }^{3} \mathrm{H}\right]$ uridine, $\left[{ }^{3} \mathrm{H}\right]$ leucine and $\left[{ }^{3} \mathrm{H}\right]-\mathrm{N}$-acetylglucosamine, respectively) was measured using $\mathrm{S}$. aureus ATCC 29213 (methicillin-sensitive S. aureus (MSSA)). DNA intercalation was examined using a commercially available kit. In the absence of DNA intercalation, relaxed plasmid migrates as a series of discrete topomers, with a characteristic ladder pattern. In contrast, intercalation results in a change in linking number, resulting in a change in the migration pattern. 'SC', supercoiled; 'Rel', relaxed.

model even when the compound was administered after infection. Given this initial demonstration of in vivo activity, investigation of additional dosing regimens for this compound and infection models (cutaneous, pulmonary, systemic) is now ongoing.

Anthracimycin demonstrated rapid killing kinetics against MRSA, although the postantibiotic effect was quite negligible. It is possible that very little of the compound remains associated with the bacteria once it is removed from the media or that anthracimycin is highly susceptible to degradation, and future studies will address anthracimycin stability. Interestingly, our in vitro data suggest that anthracimycin was still able to exert effects on MRSA at

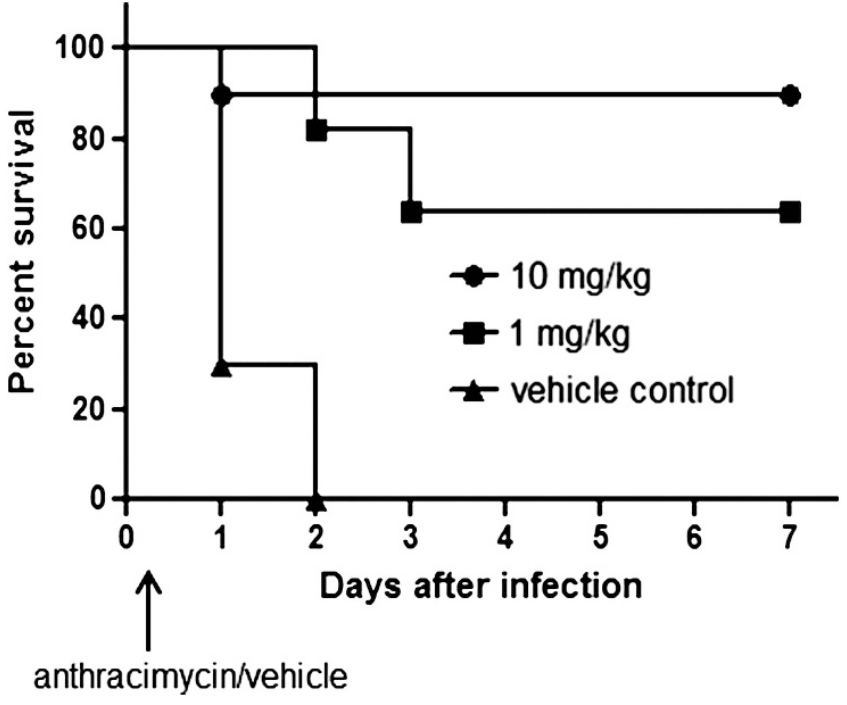

Figure 5 Anthracimycin protection of mice from methicillin-resistant $S$. aureus (MRSA) mortality. Female CD1 mice were infected intraperitoneally $\left(\sim 10^{9}\right.$ CFUs per mouse) with MRSA strain Sanger 252 and treated i.p. $1 \mathrm{~h}$ after infection with either anthracimycin ( 1 or $10 \mathrm{mg} \mathrm{kg}^{-1}$ ) or vehicle control ( $n=10$ mice per group). Survival was monitored over 7 days. These survival data are from one representative study that was repeated a total of two times.

concentrations below its MIC. We found a marked increase in lag phase when anthracimycin was present at $1 / 16 \times \mathrm{MIC}$; no similar effects were observed with the cell wall agent vancomycin up to $1 / 2 \times$ its MIC against MRSA. Notably, sub-MIC anthracimycin was able to potentiate the effects of the human cathelicidin LL-37 on MRSA growth. In these assays growth of MRSA was observed during $24 \mathrm{~h}$ in the presence of $1 / 2 \times$ MIC LL-37 or $1 / 4 \times$ MIC anthracimycin; however, no growth over the same time period was observed when the two were combined at those respective concentrations. It is possible that anthracimycin may act in synergy with LL-37 to hinder MRSA growth. This sensitization is not without precedent; for example, at well below its MIC, ampicillin has previously been shown to sensitize ampicillin-resistant Enterococcus to LL-37-mediated killing. ${ }^{12}$ Additional studies will continue to investigate anthracimycin interactions with the host immune system, including with other host-derived antimicrobial peptides. 
In summary, our initial in vitro and in vivo studies suggest that the anthracimycin scaffold may serve as a viable lead for a medicinal chemistry effort to achieve a useful new MRSA therapeutic.

\section{ACKNOWLEDGEMENTS}

This work was supported by the National Institutes of Health (NIH) Training Program in Marine Biotechnology (T32 GM067550), a Ruth L Kirschstein National Research Service Award (NRSA) from National Institutes of Health Grants (5 F31 GM090658-02) and by National Institutes of Health Grant GM084350 to WF and VN. Part of this work was funded by the Transformational Medical Technologies program contract HDTRA1-10-C-004 from the Department of Defense Chemical and Biological Defense program through the Defense Threat Reduction Agency. We gratefully acknowledge the in vivo technical expertise of Joshua Olson.

1 David, M. Z., Medvedev, S., Hohmann, S. F., Ewigman, B. \& Daum, R. S. Increasing burden of methicillin-resistant Staphylococcus aureus hospitalizations at US academic medical centers, 2003-2008. Infect. Control. Hosp. Epidemiol. 33, 782-789 (2012).

2 Klein, E. Y., Sun, L., Smith, D. L. \& Laxminarayan, R. The changing epidemiology of methicillin-resistant Staphylococcus aureus in the United States: a national observational study. Am. J. Epidemiol. 177, 666-674 (2013).
3 Uhlemann, A. C., Otto, M., Lowy, F. D. \& Deleo, F. R. Evolution of community- and healthcare-associated methicillin-resistant Staphylococcus aureus. Infect. Genet. Evol. 21, 563-574 (2013).

4 Oh, D. C., Kauffman, C. A., Jensen, P. R. \& Fenical, W. Induced production of emericellamides $\mathrm{A}$ and $\mathrm{B}$ from the marine-derived fungus Emericella sp. in competing co-culture. J. Nat. Prod. 70, 515-520 (2007).

5 Hughes, C. C., Prieto-Davo, A., Jensen, P. R. \& Fenical, W. The marinopyrroles, antibiotics of an unprecedented structure class from a marine Streptomyces sp. Org. Lett. 10, 629-631 (2008).

6 Asolkar, R. N., Kirkland, T. N., Jensen, P. R. \& Fenical, W. Arenimycin, an antibiotic effective against rifampin- and methicillin-resistant Staphylococcus aureus from the marine actinomycete Salinispora arenicola. J. Antibiot. 63, 37-39 (2010).

7 Sun, P. et al. Fijimycins A-C, three antibacterial etamycin-class depsipeptides from a marine-derived Streptomyces sp. Bioorg. Med. Chem. 19, 6557-6562 (2011).

8 Sakoulas, G. et al. Novel bacterial metabolite merochlorin A demonstrates in vitro activity against multi-drug resistant methicillin-resistant Staphylococcus aureus. PLoS One 7, e29439 (2012).

9 Jang, K. H. et al. Anthracimycin, a potent anthrax antibiotic from a marine-derived actinomycete. Angew. Chem. Int. Ed. Engl. 52, 7822-7824 (2013).

10 Haste, N. M. et al. Pharmacological properties of the marine natural product marinopyrrole A against methicillin-resistant Staphylococcus aureus. Antimicrob. Agents Chemother. 55, 3305-3312 (2011).

11 Cunningham, M. L., Kwan, B. P., Nelson, K. J., Bensen, D. C. \& Shaw, K. J. Distinguishing on-target versus off-target activity in early antibacterial drug discovery using a macromolecular synthesis assay. J. Biomol. Screen. 18, 1018-1026 (2013).

12 Sakoulas, G. et al. Ampicillin enhances daptomycin- and cationic host defense peptide-mediated killing of ampicillin- and vancomycin-resistant Enterococcus faecium. Antimicrob. Agents Chemother. 56, 838-844 (2012). 